\title{
Genotype-Phenotype Associations in Relation to Evolution of Sperm Form and Function
}

\author{
Eduardo Roldan
}

Males exhibit an enormous diversity in reproductive traits. For example, they display wide variations in testes mass relative to body mass, testis architecture and kinetics of the spermatogenic cycle. These differences translate into considerable variations in relative sperm numbers. Males also show wide divergence in the morphology, size and function of spermatozoa. Sperm numbers and sperm design, which are key determinants of fertility, are likely to be under the influence of selective forces such as sperm competition and modes of gamete transport and fertilization. A general response to sperm competition is an increase in the number of sperm produced and transferred to the female tract. An overall improvement of sperm quality (e.g., high percentages of motile and normal spermatozoa) is also widely observed. Moreover, sperm swimming velocity, which is crucial to negotiate barriers in the female tract, reaches the site of fertilization and penetrates ovum vestments, is strongly influenced by the intensity of sperm competition. The velocity of spermatozoa is also affected by factors such as the shape of the sperm head, sperm dimensions (the longer the sperm, the faster their speed) and ATP levels (required to fuel cell propulsion). Genes coding for proteins involved in reproduction are also under strong selective pressure. Among these genes, protamines are important for chromatin condensation and, thus, for the determination of head morphology. Rodents and primates have two types of protamines (PRM1 and PRM2) and the proportion between them seems crucial for normal sperm formation and function. Sperm competition influences the evolution and regulation of these genes but in a very complex way.

\footnotetext{
E. Roldan $(\bowtie)$

Reproductive Ecology and Biology Group, Department of Biodiversity and Evolutionary

Biology, Museo Nacional de Ciencias Naturales (CSIC), Madrid, Spain

e-mail: roldane@mncn.csic.es
} 